\title{
Selection in cohorts and pattern of causes of death
}

\author{
H. Th. WAALER \\ From the Norwegian Research Council for Science and the Humanities
}

The pattern of causes of death is constantly changing over time. Some diseases are in retreat, some are advancing. Epidemiologists look for causes behind the changed pattern and, occasionally, they find solutions in environmental factors, but in this note I suggest that general demographic dynamics might be sufficient to explain the impact of external factors.

A newborn cohort is under constant reduction until it is completely extinct a century later. This reduction is due to death and also in limited geographical areas to emigration.

One might reasonably assume that the resultant selection of survivors is not random, but that it is a systematic process related among other things to the genetic characteristics. A specific cause of death will hit individuals genetically predisposed to this disease and so the cohort survivors will experience a continuous change in their genetic composition. Two cohorts which experience different reductions from death and emigration will deviate over time in their genetic structure and thus within the same age group they will tend to have different causes of death.

The question is whether this mechanism, which undoubtedly exists, is of a potentially relevant magnitude. I shall here present evidence that such cohort selection during the latest decade has been considerable in Norway.

The observations are from official Norwegian demographic statistics, but the phenomenon described will be similar in populations with similar demographic changes in mortality and emigration. I have followed decennial cohorts born over the last one and a half centuries and observed the number of survivors in the age groups 30-39, 40-49, and 50-59. For two cohorts, born in the decades $1880-89$ and 1940-49, I have followed the survivors from death and emigration up to 1980 . The results are given in the Figure.

If one compares the survivors aged 40-49 in 1930 and in 1980 the difference is striking. In 1930 the cohort had been reduced to $50 \%$ of its original size, whereas in 1980 there were close to $89 \%$ still living in the country in this age group.

The difference of $39 \%$ represents individuals who had been lost from the older cohort relative to the later cohort. The causes of death of these $39 \%$ were

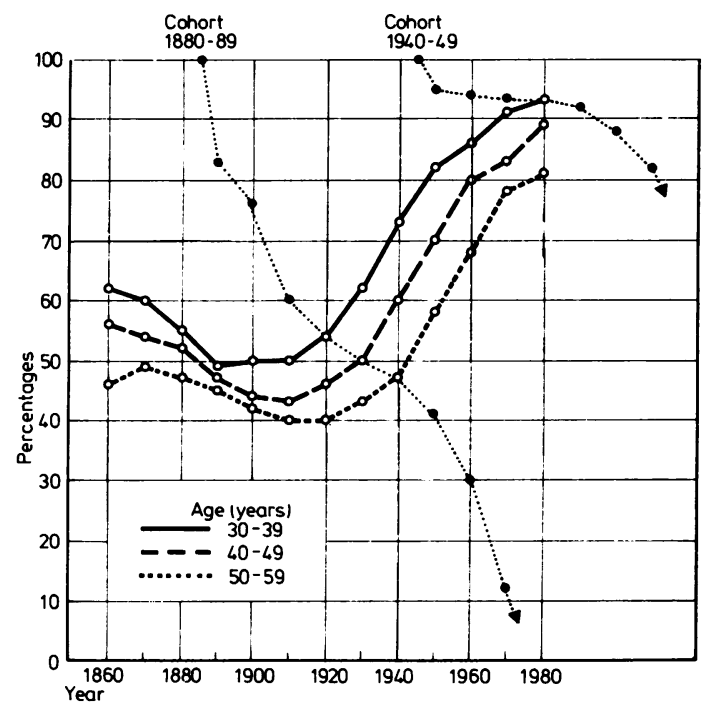

Figure Survival of cohorts in Norway.

primarily infant mortality, tuberculosis, and pneumonia, and this cohort was also affected by considerable emigration around the turn of the century.

This $39 \%$ fraction of a cohort must be different from the rest in genetic characteristics. And being of this magnitude it is likely that the two cohorts presented in the Figure will demonstrate different patterns of causes of death.

It is tempting to postulate that those individuals previously dying from the causes mentioned are those particularly subjected to cardiovascular deaths in the later cohorts, thus explaining the epidemic rise in ischaemic heart disease in the middle-aged group from 1950 to 1970 . But I admit that I have no independent evidence to that effect.

Reprints from Dr. Hans Th. Waaler, Unit for Health Services Research, the Norwegian Research Council for Science and the Humanities, Fr. Stangsgt 11/13, Oslo 2, Norway. 